Rev. Mat. Iberoamericana 19 (2003), 23-55

\title{
Hermite functions and uncertainty principles for the Fourier and the windowed Fourier transforms
}

\author{
Aline Bonami, Bruno Demange and Philippe Jaming
}

\begin{abstract}
We extend an uncertainty principle due to Beurling into a characterization of Hermite functions. More precisely, all functions $f$ on $\mathbb{R}^{d}$ which may be written as $P(x) \exp (-\langle A x, x\rangle)$, with $A$ a real symmetric definite positive matrix, are characterized by integrability conditions on the product $f(x) \widehat{f}(y)$. We then obtain similar results for the windowed Fourier transform (also known, up to elementary changes of functions, as the radar ambiguity function or the Wigner transform). We complete the paper with a sharp version of Heisenberg's inequality for this transform.
\end{abstract}

\section{Introduction and Notations.}

Uncertainty principles state that a function and its Fourier transform cannot be simultaneously sharply localized. To be more precise, let $d \geq 1$ be the dimension, and let us denote by $\langle.,$.$\rangle the scalar product and by \|$.$\| the$ Euclidean norm on $\mathbb{R}^{d}$. Then, for $f \in L^{2}\left(\mathbb{R}^{d}\right)$, define the Fourier transform of $f$ by

$$
\widehat{f}(y)=\int_{\mathbb{R}^{d}} f(t) e^{-2 i \pi\langle t, y\rangle} d t .
$$

The most famous uncertainty principle, due to Heisenberg and Weil, can be stated in the following directional version:

Heisenberg's inequality. Let $i=1, \ldots, d$ and $f \in L^{2}\left(\mathbb{R}^{d}\right)$. Then

$$
\inf _{a \in \mathbb{R}}\left(\int_{\mathbb{R}^{d}}\left(x_{i}-a\right)^{2}|f(x)|^{2} d x\right) \inf _{b \in \mathbb{R}}\left(\int_{\mathbb{R}^{d}}\left(\xi_{i}-b\right)^{2}|\widehat{f}(\xi)|^{2} d \xi\right) \geq \frac{\|f\|_{L^{2}}^{4}}{16 \pi^{2}}
$$

2000 Mathematics Subject Classification: 42B10; 32A15; $94 \mathrm{~A} 12$

Keywords: Uncertainty principles; short-time Fourier transform; windowed Fourier transform; Gabor transform; ambiguity function; Wigner transform; spectrogramm 
Moreover (1.1) is an equality if and only if $f$ is of the form

$$
f(x)=C\left(x_{1}, \ldots, x_{i-1}, x_{i+1}, \ldots, x_{n}\right) e^{-2 i \pi b x_{i}} e^{-\alpha\left(x_{i}-a\right)^{2}}
$$

where $C$ is a function in $L^{2}\left(\mathbb{R}^{d-1}\right), \alpha>0$, and $a$ and $b$ are real constants for which the two infimums in (1.1) are realized.

The usual non-directional uncertainty principle follows easily from this one. We refer to the recent survey articles by Folland and Sitaram [11] and Dembo, Cover and Thomas [9] as well as the book of Havin and Jöricke [17] for various uncertainty principles of different nature which may be found in the literature. One theorem stated in [11] is due to Beurling. Its proof has been written much later by Hörmander in [18]. Our first aim is to weaken the assumptions so that non zero solutions given by Hermite functions are also possible. More precisely, we will prove the following theorem :

Theorem 1.1 (Beurling-Hörmander type) Let $f \in L^{2}\left(\mathbb{R}^{d}\right)$ and $N \geq 0$. Then

$$
\iint_{\mathbb{R}^{d} \times \mathbb{R}^{d}} \frac{|f(x)||\widehat{f}(y)|}{(1+\|x\|+\|y\|)^{N}} e^{2 \pi|\langle x, y\rangle|} d x d y<+\infty
$$

if and only if $f$ may be written as

$$
f(x)=P(x) e^{-\pi\langle A x, x\rangle},
$$

where $A$ is a real positive definite symmetric matrix and $P$ is a polynomial of degree $<\frac{N-d}{2}$.

In particular, for $N \leq d$, the function $f$ is identically 0 . BeurlingHörmander's original theorem is the above theorem for $d=1$ and $N=0$. An extension to $d \geq 1$ but still $N=0$ has been given, first by S.C. Bagchi and S. K. Ray in [2] in a weaker form, then very recently by S. K. Ray and E. Naranayan in the present form. Their proof, which relies on the one dimensional case, uses Radon transform [25].

Let us remark that the idea of characterizing Hermite functions by pointwise vanishing at infinity, for both the function and its Fourier transform, goes back to Hardy. Indeed, such a characterization is contained in Hardy's original theorem [16], (though textbooks usually restrict attention to the characterization of Gaussian functions in Hardy's Theorem).

One may also consult [27] for extensions. The proof given here with integrability conditions uses new ingredients compared to the original proof of Hörmander [18]. At the same time, it simplifies Hörmander's argument 
for the case $N=0, d=1$, in such a way that the proof can now be given in any textbook on Fourier Analysis. We give this last one in the Appendix, since it may be useful in this context.

The previous theorem has as an immediate corollary the following characterization.

Corollary 1.2 A function $f \in L^{2}\left(\mathbb{R}^{d}\right)$ may be written as

$$
f(x)=P(x) e^{-\pi\langle A x, x\rangle},
$$

with $A$ a real positive definite symmetric matrix and $P$ a polynomial, if and only if the function

$$
f(x) \widehat{f}(y) e^{2 \pi|\langle x, y\rangle|}
$$

is slowly increasing on $\mathbb{R}^{d} \times \mathbb{R}^{d}$.

As an easy consequence of the previous theorem, we also deduce the following corollary, which generalizes the Cowling-Price uncertainty principle (see [8]).

Theorem 1.3 (Cowling-Price type) Let $N \geq 0$. Assume that $f \in L^{2}\left(\mathbb{R}^{d}\right)$ satisfies

$$
\int_{\mathbb{R}^{d}}|f(x)| \frac{e^{\pi a\left|x_{j}\right|^{2}}}{\left(1+\left|x_{j}\right|\right)^{N}} d x<+\infty \text { and } \int_{\mathbb{R}^{d}}|\widehat{f}(y)| \frac{e^{\pi b\left|y_{j}\right|^{2}}}{\left(1+\left|y_{j}\right|\right)^{N}} d y<+\infty
$$

for $j=1, \ldots, d$ and for some positive constants $a$ and $b$ with $a b=1$. Then $f(x)=P(x) e^{-a\|x\|^{2}}$ for some polynomial $P$.

The Cowling-Price type theorem is given in [8] for $d=1, N=0$, and with $p$-th powers as well. This last extension is a trivial consequence of Hölder's inequality once $N$ is allowed to take positive values. For higher dimension, we use a trick due to S.C. Bagchi and S. K. Ray to have only directional conditions. For $p=\infty$, this gives a directional Hardy type theorem.

It is remarked in [18], as well as in [2], that a theorem of BeurlingHörmander type implies also a theorem of Morgan type. But the constant that one obtains when doing this is not the best one. In fact, the best constant has been given by G. Morgan in [24] which is contemporary of the paper by Hardy. One may also consult the work of Nazarov [26] as well as [17] for more comments on the work of Morgan. We give here integrability conditions. 
Theorem 1.4 (Morgan type) Let $1<p<2$, and let $q$ be the conjugate exponent. Assume that $f \in L^{2}\left(\mathbb{R}^{d}\right)$ satisfies

$$
\int_{\mathbb{R}^{d}}|f(x)| e^{2 \pi \frac{a^{p}}{p}\left|x_{j}\right|^{p}} d x<+\infty \quad \text { and } \quad \int_{\mathbb{R}^{d}}|\widehat{f}(y)| e^{2 \pi \frac{b^{q}}{q}\left|y_{j}\right|^{q}} d y<+\infty
$$

for some $j=1, \ldots, d$ and for some positive constants $a$ and $b$. Then $f=0$ if $a b>\left|\cos \left(\frac{p \pi}{2}\right)\right|^{1 / p}$.

If $a b<\left|\cos \left(\frac{p \pi}{2}\right)\right|^{1 / p}$, one may find a dense subset of functions which satisfy the above conditions for all $j$.

From Theorem 1.1, one only gets the first result for $a b \geq 1$, which is clearly much weaker. Such a theorem is sometimes called of "Gel'fandShilov type", as these authors have studied similar conditions in their work on the classes of distributions that have a Fourier transform.

One way one may hope to overcome the lack of localisation is to use the windowed Fourier transform, also known as the (continuous) Gabor transform or the short-time Fourier transform. To be more precise, fix $v \in L^{2}\left(\mathbb{R}^{d}\right)$, the "window", and define for $u \in L^{2}\left(\mathbb{R}^{d}\right)$ :

$$
S_{v} u(x, y)=\widehat{u \bar{v}(.-x)}(y)=\int_{\mathbb{R}^{d}} u(t) \overline{v(t-x)} e^{-2 i \pi\langle t, y\rangle} d t .
$$

This transform occurs also in several other forms, for example $\left|S_{v} u\right|^{2}$ is known as a spectrogram. For sake of symmetry in $u$ and $v$, we rather focus on the radar ambiguity function defined for $u, v \in L^{2}\left(\mathbb{R}^{d}\right)$ by

$$
A(u, v)(x, y)=\int_{\mathbb{R}^{d}} u\left(t+\frac{x}{2}\right) \overline{v\left(t-\frac{x}{2}\right)} e^{-2 i \pi\langle y, t\rangle} d t
$$

Since $|A(u, v)|=\left|S_{v} u\right|$, there will be no loss in doing so. We refer the reader to $[1],[7],[6]$ and the references there for the way these functions occur in signal processing, and their basic properties.

Finally, $W(u, v)$, the Fourier transform of $A(u, v)$ in $\mathbb{R}^{2 d}$ is known in quantum mechanics and in the PDE community as the Wigner transform or Wigner distribution. Since

$$
W(u, v)(x, y)=\int_{\mathbb{R}^{d}} u\left(x+\frac{t}{2}\right) \overline{v\left(x-\frac{t}{2}\right)} e^{2 i \pi\langle y, t\rangle} d t
$$

$W(u, v)$ is also related to $A(u, v)$ by

$$
W(u, v)(x, y)=2^{d} A(u, Z v)(2 x,-2 y)
$$


where $Z v(x)=v(-x)$. So again, all results stated here can be restated in terms of the Wigner transform.

Our second aim here is to extend uncertainty principles to the radar ambiguity functions. In particular, we will show that $A(u, v)$ satisfies theorems of Cowling-Price type on one side, of Morgan type on the other one. Both results are sharp, with the same characterization of the Hermite functions in the first case. Let us mention that the Hardy's theorem has been extended in this context by K. Gröchenig and G. Zimmermann in [15].

We also give a version of Heisenberg's inequality for $A(u, v)$ that is stronger than a previous version by A.J.E.M. Janssen ([21], see also [10]). The one dimensional case for the Wigner transform $W(u, u)$ can be found in [3]. This Heisenberg's inequality may be stated in the following matricial form.

Theorem 1.5 Assume that $u$ and $v$ be in $L^{2}\left(\mathbb{R}^{d}\right)$, with $\|u\|_{L^{2}}\|v\|_{L^{2}}=1$, and

$$
\int_{R^{d}}\|x\|^{2}\left(|u|^{2}+|v|^{2}+|\widehat{u}|^{2}+|\widehat{v}|^{2}\right) d x<\infty .
$$

Let $(X, Y)$ be a random vector with probability density given on $\mathbb{R}^{d} \times \mathbb{R}^{d}$ by the function $|A(u, v)|^{2}$. Then $X$ and $Y$ are not correlated, and their two covariance matrices are such that

$$
4 \pi^{2} V(X)-V(Y)^{-1}
$$

is semi-positive definite. Moreover, if it is the zero matrix, then $u$ and $v$ are Gaussian functions.

In Radar Theory, for which $d=1$, the couple $(X, Y)$ may be given a physical meaning: its first component is related to the distance to the target, the second to its velocity. So the variance gives the quadratic error when estimating the distance or the velocity by the corresponding mean.

A different problem consists in minimizing the same quantity $A(u, v)$ for a fixed "window" $v$, or more generally to consider uncertainty principles in terms of a function $u$ and its windowed Fourier transform $A(u, v)$. Such problems have been considered by E. Wilczock [29]. Best results follow from ours only when the window is Gaussian.

The article is organized as follows: the next section is devoted to the proof of Theorem 1.1, whereas in section 3, we consider the other mentioned uncertainty principles for the Fourier transform. In section 4, we recall some basic properties of the ambiguity functions, pursuing in section 5 with the Heisenberg inequality for these functions. Section 6 is devoted to the extension of uncertainty principles to ambiguity functions. 
28 A. Bonami, B. Demange and P. Jaming

\section{Generalization of Beurling-Hörmander's theorem.}

The statement of Theorem 1.1 is divided into two propositions. The first one is elementary.

Proposition 2.1 Let $f \in L^{2}\left(\mathbb{R}^{d}\right)$ be a function of the form

$$
f(x)=P(x) e^{-\pi\langle(A+i B) x, x\rangle},
$$

with $A$ and $B$ two real symmetric matrices and $P$ a polynomial. Then the matrix $A$ is positive definite. Moreover, the three following conditions are equivalent:

$$
\begin{aligned}
& B=0 \text { and } \operatorname{deg}(P)<\frac{N-d}{2} ; \\
& \iint_{\mathbb{R}^{d} \times \mathbb{R}^{d}} \frac{|f(x) \| \widehat{f}(y)|}{(1+\|x\|+\|y\|)^{N}} e^{2 \pi|\langle x, y\rangle|} d x d y<+\infty \\
& \iint_{\mathbb{R}^{d} \times \mathbb{R}^{d}} \frac{|f(x)||\widehat{f}(y)|}{(1+\|x\|)^{\frac{N}{2}}(1+\|y\|)^{\frac{N}{2}}} e^{2 \pi|\langle x, y\rangle|} d x d y<+\infty .
\end{aligned}
$$

Proof. The fact that $A$ is positive definite is elementary. Then, after a change of variables, we may assume that $A$ is the identity matrix, so that $f$ may be written as $P(x) e^{-\pi\|x\|^{2}} e^{-i \pi\langle B x, x\rangle}$. The Fourier transform of $f$ may be written as $Q(y) e^{-\pi\left\langle(I+i B)^{-1} y, y\right\rangle}$, with $\operatorname{deg}(P)=\operatorname{deg}(Q)=n$.

To prove that $(i)$ implies $(i i i)$, it is sufficient to prove that, for $\alpha>d / 2$, we have the inequality

$$
\iint_{\mathbb{R}^{d} \times \mathbb{R}^{d}}(1+\|x\|)^{-\alpha}(1+\|y\|)^{-\alpha} e^{-\pi\|x-y\|^{2}} d x d y<+\infty .
$$

But this integral is twice the integral on the subset where $\|x\| \leq\|y\|$. So it is bounded by

$$
2 \int_{\mathbb{R}^{d}}\left[(1+\|x\|)^{-2 \alpha} \times \int_{\mathbb{R}^{d}} e^{-\pi\|x-y\|^{2}} d y\right] d x<+\infty
$$

which allows to conclude.

It is clear that $(i i i)$ implies $(i i)$ for all functions $f$. Let us now prove that $(i i)$ implies $(i)$. First, writing $(I+i B)^{-1}=(I-i B)\left(I+B^{2}\right)^{-1}$, it is immediate that the integrability on $f(x) \widehat{f}(y)$ implies that the homogeneous polynomial $\|x\|^{2}+\left\langle\left(I+B^{2}\right)^{-1} y, y\right\rangle-2\langle x, y\rangle$ is non negative, which implies that $B=0$. Now, let $P_{n}$ and $Q_{n}$ be the homogeneous terms of maximal 
degree of the polynomials $P$ and $Q$. There exists $x^{(0)} \in \mathbb{R}^{d}$ of norm 1 such that $P_{n}\left(x^{(0)}\right) Q_{n}\left(x^{(0)}\right)$ is different from 0 . We call $\Gamma_{\varepsilon}$ the cone under consideration. It is obtained as the image of the cone

$$
\left\{x=\left(x_{1}, \ldots, x_{d}\right) ;\left(x_{2}^{2}+\cdots+x_{d}^{2}\right)^{\frac{1}{2}}<\varepsilon x_{1}\right\}
$$

under a rotation which maps the point $(1,0, \ldots, 0)$ to $x^{(0)}$. Then, for $\varepsilon<1$ small enough, there exists a constant $c$ such that, for $x, y \in \Gamma_{\varepsilon}$, one has the inequality

$$
\left|P_{n}(x)\right|\left|Q_{n}(y)\right| \geq c\|x\|^{n}\|y\|^{n}
$$

The same inequality is valid for $P$ and $Q$ for $x$ and $y$ large, which implies that

$$
\iint_{\Gamma_{\varepsilon} \times \Gamma_{\varepsilon}} \frac{\|x\|^{n}\|y\|^{n}}{(1+\|x\|+\|y\|)^{N}} e^{-\pi\|x-y\|^{2}} d x d y<+\infty .
$$

We remark that if $x \in \Gamma_{\varepsilon}$ then $-\Gamma_{\varepsilon} \subset x-\Gamma_{\varepsilon}$. So, a fortiori, we have that

$$
\iint_{\Gamma_{\varepsilon} \times\left(-\Gamma_{\varepsilon}\right)} \frac{\|x\|^{n}\|t+x\|^{n}}{(1+\|x\|+\|t+x\|)^{N}} e^{-\pi\|t\|^{2}} d x d t<+\infty .
$$

We know, using Fubini's theorem, that there exists $t$ for which

$$
\int_{\Gamma_{\varepsilon}} \frac{\|x\|^{n}\|t+x\|^{n}}{(1+\|x\|+\|t+x\|)^{N}} d x<+\infty
$$

which proves that $N-2 n>d$.

We have written here the equivalence between Conditions ( $i i)$ and (iii) to remark that Condition (iii) could be written in place of (1.2) in Theorem 1.1.

The next proposition is much deeper.

Proposition 2.2 Let $f \in L^{2}\left(\mathbb{R}^{d}\right)$. If, for some positive integer $N$,

$$
\iint_{\mathbb{R}^{d} \times \mathbb{R}^{d}} \frac{|f(x)||\widehat{f}(y)|}{(1+\|x\|+\|y\|)^{N}} e^{2 \pi|\langle x, y\rangle|} d x d y<+\infty
$$

then $f$ may be written as

$$
f(x)=P(x) e^{-\pi\langle(A+i B) x, x\rangle},
$$

where $A$ and $B$ are two symmetric matrices and $P$ is a polynomial. 
30 A. Bonami, B. Demange and P. Jaming

Proof. We may assume that $f \neq 0$.

First step. Both $f$ and $\widehat{f}$ are in $L^{1}\left(\mathbb{R}^{d}\right)$.

For almost every $y$,

$$
|\widehat{f}(y)| \int_{\mathbb{R}^{d}} \frac{|f(x)|}{(1+\|x\|)^{N}} e^{2 \pi|\langle x, y\rangle|} d x<+\infty .
$$

As $f \neq 0$, the set of all $y$ 's such that $\widehat{f}(y) \neq 0$ has positive measure. In particular, there is a basis $y^{(1)}, \ldots, y^{(d)}$ of $\mathbb{R}^{d}$ such that, for $i=1, \ldots, d$, $\widehat{f}\left(y^{(i)}\right) \neq 0$ and

$$
\int_{\mathbb{R}^{d}} \frac{|f(x)|}{(1+\|x\|)^{N}} e^{2 \pi\left|\left\langle x, y^{(i)}\right\rangle\right|} d x<+\infty .
$$

Since, clearly, there exists a constant $C$ such that

$$
(1+\|x\|)^{N} \leq C \sum_{i=1}^{d} e^{2 \pi\left|\left\langle x, y^{(i)}\right\rangle\right|},
$$

we get $f \in L^{1}\left(\mathbb{R}^{d}\right)$. Exchanging the roles of $f$ and $\widehat{f}$, we get $\widehat{f} \in L^{1}\left(\mathbb{R}^{d}\right)$.

Second step. The function $g$ defined by $\widehat{g}(y)=\widehat{f}(y) e^{-\pi\|y\|^{2}}$ satisfies the following properties (with $C$ depending only on $f$ )

$$
\begin{gathered}
\int_{\mathbb{R}^{d}}|\widehat{g}(y)| e^{\pi\|y\|^{2}} d y<\infty ; \\
|\widehat{g}(y)| \leq C e^{-\pi\|y\|^{2}} ; \\
\iint_{\mathbb{R}^{d} \times \mathbb{R}^{d}} \frac{|g(x)||\widehat{g}(y)|}{(1+\|x\|+\|y\|)^{N}} e^{2 \pi|\langle x, y\rangle|} d x d y<+\infty ; \\
\int_{\|x\| \leq R} \int_{\mathbb{R}^{d}}|g(x)||\widehat{g}(y)| e^{2 \pi|\langle x, y\rangle|} d x d y<C(1+R)^{N} .
\end{gathered}
$$

Property (2.2) is obvious from the definition of $g$ and the fact that $\widehat{f}$ is in $L^{1}\left(\mathbb{R}^{d}\right)$. As $f \in L^{1}\left(\mathbb{R}^{d}\right), \widehat{f}$ is bounded thus (2.3) is also obvious. To prove (2.4), we have

$$
\iint_{\mathbb{R}^{d} \times \mathbb{R}^{d}} \frac{|g(x)||\widehat{g}(y)|}{(1+\|x\|+\|y\|)^{N}} e^{2 \pi|\langle x, y\rangle|} d x d y \leq \iint|f(t)||\widehat{f}(y)| A(t, y) e^{2 \pi|\langle t, y\rangle|} d t d y
$$


with

$$
A(t, y)=\int \frac{e^{-\pi\|x\|^{2}-\pi\|y\|^{2}+2 \pi|\langle x, y\rangle|}}{(1+\|t-x\|+\|y\|)^{N}} d x
$$

We claim that

$$
A(t, y) \leq C(1+\|t\|+\|y\|)^{-N},
$$

which allows to conclude. Indeed, separating the cases of $\langle x, y\rangle$ being positive or negative, we get

$$
\begin{aligned}
A(t, y) & \leq \int \frac{e^{-\pi\|x-y\|^{2}}}{(1+\|t-x\|+\|y\|)^{N}} d x+\int \frac{e^{-\pi\|x+y\|^{2}}}{(1+\|t+x\|+\|y\|)^{N}} d x \\
& =I_{1}+I_{2} .
\end{aligned}
$$

As $I_{2}(t, y)=I_{1}(-t, y)$, it is enough to get a bound for $I_{1}$. Now, fix $0<c<1$ and write $B=(1+\|t\|+\|y\|)$, then

$$
I_{1} \leq \int_{\|x-y\|>c B} e^{-\pi\|x-y\|^{2}} d x+\int_{\|x-y\| \leq c B} \frac{e^{-\pi\|x-y\|^{2}}}{(1+\|t-x\|+\|y\|)^{N}} d x .
$$

We conclude directly for the first integral. For the second one, it is sufficient to note that, if $\|x-y\| \leq c(1+\|t\|+\|y\|)$, then

$$
\begin{aligned}
1+\|t-x\|+\|y\| & \geq 1+\frac{1}{2}\|t\|+\frac{1}{2}\|y\|-\frac{1}{2}\|x-y\| \\
& \geq \frac{(1-c)}{2}(1+\|t\|+\|y\|) .
\end{aligned}
$$

This completes the proof of (2.6) and (2.4).

Let us finally prove (2.5). Fix $c>2$. Then the left hand side is bounded by

$$
\begin{aligned}
& \int_{\|x\| \leq R}|g(x)|\left(\int_{\|y\|>c R}|\widehat{g}(y)| e^{2 \pi|\langle x, y\rangle|} d y+\int_{\|y\|<c R}|\widehat{g}(y)| e^{2 \pi|\langle x, y\rangle|} d y\right) d x \\
& \leq \int_{\|x\| \leq R}|g(x)|\left(\int_{\|y\|>c R} C e^{-\left(\pi-2 \frac{\pi}{c}\right)\|y\|^{2}} d y+\int_{\|y\|<c R}|\widehat{g}(y)| e^{2 \pi|\langle x, y\rangle|} d y\right) d x \\
& \leq K\|g\|_{L^{1}}+\int_{\|x\| \leq R} \int_{\|y\|<c R}|g(x) \| \widehat{g}(y)| e^{2 \pi|\langle x, y\rangle|} d x d y .
\end{aligned}
$$

Then, if we multiply and divide by $(1+\|x\|+\|y\|)^{N}$ in the integral of right side, we get the required inequality (2.5). This completes the proof of the claim. 
Third step. The function $g$ admits an holomorphic extension to $\mathbb{C}^{d}$ that is of order 2. Moreover, there exists a polynomial $R$ such that for all $z \in \mathbb{C}^{d}$, $g(z) g(i z)=R(z)$.

It follows from (2.3) and Fourier inversion that $g$ admits an holomorphic extension to $\mathbb{C}^{d}$ which we again denote by $g$. Moreover,

$$
|g(z)| \leq C e^{\pi\|z\|^{2}}
$$

with $C$ the $L^{1}$ norm of $\widehat{g}$. It follows that $g$ is of order 2 . On the other hand, for all $x \in \mathbb{R}^{d}$ and $e^{i \theta}$ of modulus 1 ,

$$
\left|g\left(e^{i \theta} x\right)\right| \leq \int_{\mathbb{R}^{d}}|\widehat{g}|(y) e^{2 \pi|\langle x, y\rangle|} d y .
$$

Let us now define a new function $G$ on $\mathbb{C}^{d}$ by:

$$
G: z \rightarrow \int_{0}^{z_{1}} \cdots \int_{0}^{z_{d}} g(u) g(i u) d u .
$$

As $g$ is entire of order 2, so is $G$. By differentiation of $G$, the proof of this step is complete once we show that $G$ is a polynomial.

To do so, we will use (2.5) and an elementary variant of PhragmènLindelhöf's principle which we recall here, and which may be found in [14]: let $\phi$ be an entire function of order 2 in the complex plane and let $\alpha \in] 0, \pi / 2[$; assume that $|\phi(z)|$ is bounded by $C(1+|z|)^{N}$ on the boundary of some angular sector $\left\{r e^{i \beta}: r \geq 0, \beta_{0} \leq \beta \leq \beta_{0}+\alpha\right\}$. Then the same bound is valid inside the angular sector (when replacing $C$ by $2^{N} C$ ).

Let us fix a vector $\xi \in \mathbb{R}^{d}$ and define the function $G_{\xi}$ on $\mathbb{C}$ by $G_{\xi}(z)=$ $G(z \xi)$. Then $G_{\xi}$ is an entire function of order 2 which has polynomial growth on $\mathbb{R}$ and on $i \mathbb{R}$ by (2.7) and (2.5). We cannot directly apply PhragmènLindelhöf's principle since we are not allowed to do so on angular sectors of angle $\pi / 2$. But, to prove that $G$ has polynomial growth in the first quadrant, it is sufficient to prove uniform estimates of this type inside all angular sectors $\left\{r e^{i \beta}: r \geq 0,0<\beta_{0} \leq \beta \leq \pi / 2\right\}$. Moreover, it is sufficient to have uniform estimates for the functions $G_{\xi}^{(\alpha)}(z)=G^{(\alpha)}(z \xi)$, with $0<\alpha<\beta_{0}$, and

$$
G^{(\alpha)}(z)=\int_{0}^{z_{1}} \cdots \int_{0}^{z_{d}} g\left(e^{-i \alpha} u\right) g(i u) d u .
$$

$G_{\xi}^{(\alpha)}$ clearly has polynomial growth on $e^{i \alpha} \mathbb{R}$ and on $i \mathbb{R}$, that is

$$
G_{\xi}^{(\alpha)}(z) \leq C(1+|x|\|\xi\|)^{N}
$$


The constant $C$, which comes from the constant in (2.5), is independent of $\alpha$. The same estimate is valid inside the angular sector by the PhragmènLindelhöf's principle, and extends to $G_{\xi}$, which we wanted to prove.

Proceeding in an analogous way in the three other quadrants, we prove that $G_{\xi}$ is an entire function with polynomial growth of order $N$, so a polynomial of degree $\leq N$. Let us now write

$$
G_{\xi}(z)=a_{0}(\xi)+\cdots+a_{N}(\xi) z^{N}
$$

Then

$$
a_{j}(\xi)=\left.\frac{1}{j !} \frac{d^{j}}{d z^{j}}(G(z \xi))\right|_{z=0}
$$

shows that $a_{j}$ is a homogeneous polynomial of degree $j$ on $\mathbb{R}^{d}$.

The entire function $G$, which is a polynomial on $\mathbb{R}^{d}$, is a polynomial. Finally,

$$
g(z) g(i z)=R(z)
$$

where $R$ is a polynomial and the proof of this step is complete.

Fourth Step. A lemma about entire functions of several variables.

We are now lead to solving the equation (2.8), where $g$ is an entire function of order 2 of $d$ variables and $R$ is a given polynomial. It is certainly well known that such functions $g$ can be written as $P(z) e^{Q(z)}$, with $Q(z)$ a polynomial of degree at most 2. Moreover, the equation implies that $Q(z)+Q(i z)=0$, so that $Q$ is homogeneous of degree 2 . So we have completed the proof, up to the study of the equation (2.8). Since we did not find a simple reference for it, we include the proof of the next lemma, which is a little more general than what we need above.

Lemma 2.3 Let $\varphi$ be an entire function of order 2 on $\mathbb{C}^{d}$ such that, on every complex line, either $\varphi$ is identically 0 or it has at most $N$ zeros. Then, there exists a polynomial $P$ with degree at most $N$ and a polynomial $Q$ with degree at most 2 such that $\varphi(z)=P(z) e^{Q(z)}$.

Proof. Without loss of generality, we may assume that $\varphi(0)$ does not vanish. Then, for $z \in \mathbb{C}^{d}, \varphi_{z}(t)=\varphi(t z)$ is a non-zero entire function of order 2 that has at most $N$ zeros. By Hadamard's factorisation theorem, for every $z \in \mathbb{C}^{d}$, there exists a polynomial $P_{z}$ with $\operatorname{deg}\left(P_{z}\right) \leq N$ and $\alpha(z), \beta(z) \in \mathbb{C}$ such that :

$$
\varphi(t z)=P_{z}(t) e^{\alpha(z) t+\beta(z) t^{2}}
$$


34 A. Bonami, B. Demange and P. Jaming

with

$$
P_{z}(t)=a_{0}(z)+\cdots+a_{N}(z) t^{N} .
$$

From the uniqueness in Hadamard's theorem, we easily see that the functions $\alpha$ and $\beta$ are homogeneous of degree 1 and 2 respectively, and $a_{j}$ is of degree $j$. We may assume that $a_{N}(z)$ is non identically zero. We have

$$
\varphi(t z) \varphi(-t z) \varphi(i t z) \varphi(-i t z)=P_{z}(t) P_{z}(-t) P_{z}(i t) P_{z}(-i t) .
$$

Differentiating (2.9) $4 N$ times with respect to $t$, and then taking $t=0$, we get that $a_{N}(z)^{4}$ is a homogeneous polynomial of degree $4 N$. Now,

$$
\begin{aligned}
\frac{d}{d t}(\varphi(t z)) \varphi(-t z) & \varphi(i t z) \varphi(-i t z) \\
= & \left(\frac{d}{d t} P_{z}(t)+(2 \beta(z) t+\alpha(z)) P_{z}(t)\right) P_{z}(i t) P_{z}(-i t)
\end{aligned}
$$

and differentiating $4 N+1$ times with respect to $t$ at $t=0$, we get that $a_{N}(z)^{4} \beta(z)$ is holomorphic. Thus $\beta(z)$ is also holomorphic, and so a homogeneous polynomial of degree 2 . An analogous proof allows to conclude that $\alpha(z)$ is a homogeneous polynomial of degree 1 . Define $Q(z)=\beta(z)+\alpha(z)$ and $P(z)=\varphi(z) e^{-Q(z)}$. We know that $P$ is holomorphic, and we have to prove that it is a polynomial. Then

$$
P(t z)=P_{z}(t)=a_{0}(z)+\cdots+a_{N}(z) t^{N} .
$$

In particular,

$$
a_{j}(z)=\left.\frac{1}{j !} \frac{d^{j}}{d t^{j}}(P(t z))\right|_{t=0}
$$

is a holomorphic function, thus a homogeneous polynomial of degree $j$. It follows that $P(z)=a_{0}(z)+\cdots+a_{N}(z)$ is a polynomial of degree $\leq N$, which we wanted to prove to conclude for this step.

We have also completed the proof of Proposition 2.2. Indeed, $g$ has the required form thus so has $f$.

Let us make a few comments on the proof. Step 3 is very much inspired, with simplifications, from the proof of Hörmander [18]. Step 2 is not contained in [18], and simplifies greatly the proof, even for $N=0$. Further, it is easy to see that if the function $\varphi$ in Lemma 2.3 is of order less than $k$, then $\varphi(z)=P(z) e^{Q(z)}$, with $P$ a polynomial of degree at most $N$ and $Q$ a polynomial of degree at most $k$.

As we said in the introduction, the reader will find separately the proof of Beurling-Hörmander's Theorem $(N=0, d=1)$ in the Appendix. 


\section{Applications to other uncertainty principles.}

Let us first mention the following immediate corollary of Theorem 1.1:

Corollary 3.1 Let $f \in L^{2}\left(\mathbb{R}^{d}\right)$.

(i) If

$$
\iint_{\mathbb{R}^{d} \times \mathbb{R}^{d}} \frac{|f(x)||\widehat{f}(y)|}{(1+\|x\|+\|y\|)^{N}} e^{2 \pi \sum_{i=1}^{d}\left|x_{i} y_{i}\right|} d x d y<+\infty
$$

then $f(x)=P(x) e^{-\sum_{i=1}^{d} \beta_{i} x_{i}^{2}}$ with $P$ a polynomial and $\beta_{i}>0$ for $i=$ $1, \ldots, d$.

(ii) If

$$
\iint_{\mathbb{R}^{d} \times \mathbb{R}^{d}} \frac{|f(x) \| \widehat{f}(y)|}{(1+\|x\|+\|y\|)^{N}} e^{2 \pi\|x\|\|y\|} d x d y<+\infty
$$

then $f(x)=P(x) e^{-\beta\|x\|^{2}}$ with $P$ a polynomial and $\beta>0$.

Proof. It is enough to see that Conditions (3.1) and (3.2) are stronger than Condition (1.2) of Theorem 1.1. Thus $f(x)=P(x) e^{-\langle A x, x\rangle}$ for some positive definite matrix $A$. A direct computation then shows that the form of the matrix $A$ imposed by Conditions (3.1) and (3.2) are respectively $A$ diagonal and $A=\beta I$.

The next proposition, which implies the Cowling-Price theorem in one dimension, follows at once from the last case.

Proposition 3.2 Let $N \geq 0$. Assume that $f \in L^{2}\left(\mathbb{R}^{d}\right)$ satisfies

$$
\int_{\mathbb{R}^{d}} \frac{|f(x)| e^{\pi a\|x\|^{2}}}{(1+\|x\|)^{N}} d x<+\infty \quad \text { and } \quad \int_{\mathbb{R}^{d}} \frac{|\widehat{f}(y)| e^{\pi b\|x\|^{2}}}{(1+\|y\|)^{N}} d y<+\infty .
$$

Then, $a b>1$ implies $f=0$. If $a b=1$, then $f(x)=P(x) e^{-\pi a\|x\|^{2}}$ for some polynomial $P$.

Proof of Theorem 1.3. For $d=1$, this is exactly Proposition 3.2. For $d>1$, we proceed as in [2] to reduce to the one-dimensional case. For almost every $x^{\prime}=\left(x_{2}, \ldots, x_{d}\right)$, the function $f_{x^{\prime}}$ defined by $f_{x^{\prime}}\left(x_{1}\right)=f\left(x_{1}, x^{\prime}\right)$ is in $L^{2}(\mathbb{R})$ and has as Fourier transform the function

$$
y_{1} \mapsto \int_{\mathbb{R}^{d-1}} \widehat{f}\left(y_{1}, y^{\prime}\right) e^{-2 \pi i\left\langle x^{\prime}, y^{\prime}\right\rangle} d y^{\prime}
$$


So, for almost every $x^{\prime}$, the function $f_{x^{\prime}}$ satisfies the assumptions of Proposition 3.2 , and $e^{\pi a\left|x_{1}\right|^{2}} f\left(x_{1}, x^{\prime}\right)$ is a polynomial of degree at most $N-2$ in the $x_{1}$ variable. The same is valid in each variable, which allows to conclude.

Let us remark that, as in [2] for the case $N=0$, it is possible to weaken the assumption when the conclusion is that $f$ vanishes. We have the immediate corollary:

Corollary 3.3 Assume that $f \in L^{2}\left(\mathbb{R}^{d}\right)$ satisfies

$$
\int_{\mathbb{R}^{d}}|f(x)| \frac{e^{\pi a\left|x_{j}\right|^{2}}}{\left(1+\left|x_{j}\right|\right)^{M}} d x<+\infty \quad \text { and } \quad \int_{\mathbb{R}^{d}}|\widehat{f}(y)| \frac{e^{\pi b\left|y_{j}\right|^{2}}}{\left(1+\left|y_{j}\right|\right)^{N}} d y<+\infty
$$

for some $j=1, \ldots, d$ and for some positive constants $a$ and $b$ with $a b=1$. If $\min \{M, N\}=1$, then $f$ is identically 0 .

Another remark is that $L^{1}$ norms may be replaced by $L^{p}$ norms for $1 \leq$ $p \leq \infty$ in the previous statements, using Hölder's inequality. In particular, we get Corollary 1.2 as well as a modification of the usual Hardy's theorem that we state now.

Proposition 3.4 (Hardy type) Let $f \in L^{2}\left(\mathbb{R}^{d}\right)$ be such that, for all $j=$ $1, \ldots, n$,

$$
|f(x)| \leq C\left(1+\left|x_{j}\right|\right)^{N} e^{-\pi a\left|x_{j}\right|^{2}} \quad \text { and } \quad|\widehat{f}(y)| \leq C\left(1+\left|y_{j}\right|\right)^{N} e^{-\pi b\left|y_{j}\right|^{2}} .
$$

We have the following implications.

1. If $a b>1$, then $f=0$.

2. If $a b=1$, then $f(x)=P(x) e^{-\pi a\|x\|^{2}}$, where $P$ is a polynomial of degree $\leq N$.

3. Else, there is a dense subspace of functions satisfying these estimates.

Proof. It is enough to notice that the assumptions of Theorem 1.3 are satisfied (for a larger value of $N$ ).

Proof of Theorem 1.4. It is sufficient to consider the one-dimensional case: for the first statement we conclude the general case from the onedimensional one as before, and to find a dense subset of functions we use tensorization. So, let us first assume that $f \in L^{2}(\mathbb{R})$ is such that

$$
\int_{\mathbb{R}}|f(x)| e^{2 \pi \frac{a^{p}}{p}|x|^{p}} d x<+\infty \quad \text { and } \quad \int_{\mathbb{R}}|\widehat{f}(y)| e^{2 \pi \frac{b^{q}}{q}|y|^{q}} d y<+\infty
$$


for some positive constants $a$ and $b$, and prove that $f$ is identically zero. Here $1<p \leq 2$, and $q$ is the conjugate exponent. It follows from the second inequality that $f$ extends to an entire function, which satisfies the inequality

$$
|f(x+i y)| \leq C e^{\frac{2 \pi}{p}\left|\frac{y}{b}\right|^{p}} .
$$

Moreover, the same inequality is valid when $f$ is replaced by its even part, or its odd part. Such a function may be written as $g\left(z^{2}\right)$, or $z g\left(z^{2}\right)$, with $g$ an entire function. One of them is non zero, and satisfies

$$
|g(x+i y)| \leq C e^{\frac{2 \pi}{p} b^{-p}|z|^{p / 2}} \text { and } \int_{\mathbb{R}}|g(x)| e^{\frac{2 \pi}{p} a^{p}|x|^{p / 2}} d x<+\infty .
$$

In the second inequality, $a$ has effectively been replaced by an arbitrarily close smaller constant, which we write a again for simplification. We then consider the function

$$
G(z)=\int_{0}^{z} g(u) d u-\int_{0}^{\infty} g(u) d u .
$$

Then $G$ is an entire function of order $p / 2$, and, for positive $x$,

$$
|G(-x)| \leq C e^{\frac{2 \pi}{p} b^{-p} x^{p / 2}} \text { and }|G(x)| \leq C e^{-\frac{2 \pi}{p} a^{p} x^{p / 2}}
$$

eventually changing the value of $b$ into an arbitrary close one. We claim that it follows that $G$ is constant, which allows to conclude. One may refer to [24] or [26] for this.

For sake of completeness, let us give a simple proof. We may assume that $2 \pi b^{-p}=p$, and $2 \pi a^{p}=p(1+\varepsilon)\left|\cos \left(\frac{p \pi}{2}\right)\right|$. Apply the Phragmèn-Lindelöf principle in the upper-half plane as well as in the lower-half-plane for the function $G(z) \exp \left(-(-z)^{p / 2}\right)$ (using the principal branch). We find that this function, which is holomorphic outside $\mathbb{R}^{+}$, is bounded. In particular, it implies that, for some $\theta \in(0,2 \pi / p-\pi)$ and for positive $x$, the quantity $G\left(e^{i(\pi \pm \theta)} x\right) G(x)$ is bounded.

Now, consider the entire function $H: z \mapsto G\left(e^{i(\pi-\theta)} z\right) G(z)$, which is entire of order $p / 2$ and bounded on the boundary of the sector $\Gamma=\left\{x e^{i \alpha}\right.$ : $x \geq 0,-\pi+\theta \leq \alpha \leq 0\}$. Thus, applying the Phragmèn-Lindelöf principle to $H$, both on the inside and on the outside of $\Gamma$, one gets that $H$ is bounded, thus constant. But $H$ is the product of $G$ and of $z \mapsto G\left(e^{i(\pi-\theta)} z\right)$ which are both entire functions of order $\leq p / 2<2$. As they have no zeros, they are either constant or of the form $e^{a z}$. The later case is clearly excluded, so $G$ is constant as claimed. 
Let us now prove that, for $a b<\left|\cos \left(\frac{p \pi}{2}\right)\right|^{\frac{1}{p}}$, there is a dense subset of functions $f$ such that

$$
|f(x)| \leq C e^{-\frac{2 \pi}{p} a^{p}|x|^{p}} \text { and }|\widehat{f}(y)| \leq C e^{-\frac{2 \pi}{q} b^{q}|y|^{q}} .
$$

Since this set of functions is stable under multiplications by $e^{2 \pi i y_{0} x}$ (changing $b$ into an arbitrarily close smaller constant), we see immediately that it is dense, unless it reduces to 0 . Indeed, if $f$ is such a non zero function and $\phi \in L^{2}(\mathbb{R})$ is orthogonal to all functions $e^{2 \pi i y_{0} x} f$, then $\bar{f} \phi$ is identically 0 . Since $f$ is analytic, it means that $\phi$ is 0 . But the existence of such a function has been given by G. Morgan in [24] (see also [26]). This finishes the proof of Theorem 1.4.

G. Morgan gives examples of non zero functions which may be used for $a b=\left|\cos \left(\frac{p \pi}{2}\right)\right|^{\frac{1}{p}}$. Nevertheless, it leaves open the complete description of the possible functions, except for the case when $p=2$, for which the previous theorems give precise information.

\section{Properties of the ambiguity function.}

For sake of self-containedness, let us recall here a few properties of the ambiguity function that we may use in the sequel. They can be found in [1], [28].

Lemma 4.1 Let $u, v$ in $L^{2}\left(\mathbb{R}^{d}\right)$. For $a, \omega \in \mathbb{R}^{d}, \lambda>0$ define

$$
S(a) u(t)=u(t-a), \quad M(\omega) u(t)=e^{2 i \pi \omega t} u(t), \quad D_{\lambda} u(t)=\lambda^{\frac{d}{2}} u(\lambda t)
$$

and recall that we defined $Z u(t)=u(-t)$. Then

(i) $A(S(a) u, S(b) v)(x, y)=e^{-i \pi\langle a+b, y\rangle} A(u, v)(x+b-a, y)$,

(ii) $A\left(M\left(\omega_{1}\right) u, M\left(\omega_{2}\right) v\right)(x, y)=e^{i \pi\left\langle\omega_{1}+\omega_{2}, x\right\rangle} A(u, v)\left(x, y+\omega_{2}-\omega_{1}\right)$,

(iii) $A\left(D_{\lambda} u, D_{\lambda} v\right)(x, y)=A(u, v)\left(\lambda x, \frac{y}{\lambda}\right)$

(iv) $A(Z u, Z v)(x, y)=\overline{A(v, u)(x, y)}$,

(v) $A(\widehat{u}, \widehat{v})(x, y)=A(u, v)(-y,-x)$ and $A(u, v)(x, y)=\overline{A(v, u)(-x,-y)}$. 
Lemma 4.2 Let $u, v \in L^{2}\left(\mathbb{R}^{d}\right)$. Then $A(u, v)$ is continuous on $\mathbb{R}^{2 d}$ and $A(u, v) \in L^{2}\left(\mathbb{R}^{2 d}\right)$. Further,

$$
\|A(u, v)\|_{L^{2}\left(\mathbb{R}^{2 d}\right)}=\|u\|_{L^{2}\left(\mathbb{R}^{d}\right)}\|v\|_{L^{2}\left(\mathbb{R}^{d}\right)} .
$$

Proof. This fact is also well known, however to help the reader to get familiar with our notation, let us recall the proof of the last assertion (see [1], [28]). If $u$ and $v$ are fixed, we will write

$$
h_{x}(t)=u\left(t+\frac{x}{2}\right) \overline{v\left(t-\frac{x}{2}\right)} .
$$

The change of variables

$$
\xi=t-\frac{x}{2} \quad \text { and } \quad \eta=t+\frac{x}{2}
$$

gives

$$
\iint\left|h_{x}(t)\right|^{2} d t d x=\iint|u(\eta)|^{2}|v(\xi)|^{2} d \eta d \xi=\|u\|_{L^{2}\left(\mathbb{R}^{d}\right)}^{2}\|v\|_{L^{2}\left(\mathbb{R}^{d}\right)}^{2} .
$$

In particular, for almost every $x$, the integral with respect to $t$ is finite, i.e $h_{x} \in L^{2}$. Noticing that $A(u)(x, y)=\widehat{h_{x}}(y)$, and using Parseval's formula we obtain

$$
\begin{aligned}
\int\left(\int|A(u, v)(x, y)|^{2} d x\right) d y & =\int\left(\int\left|\widehat{h_{x}}(y)\right|^{2} d y\right) d x \\
& =\|u\|_{L^{2}\left(\mathbb{R}^{d}\right)}^{2}\|v\|_{L^{2}\left(\mathbb{R}^{d}\right)}^{2}
\end{aligned}
$$

which completes the proof.

Finally, we will also need the following lemma from [19], [22]:

Lemma 4.3 Let $u, v, w \in L^{2}\left(\mathbb{R}^{d}\right)$. Then, for every $x, y \in \mathbb{R}^{d}$,

$$
\begin{aligned}
\int_{\mathbb{R}^{2 d}} A(u, v)(s, t) \overline{A(v, w)(s, t)} & e^{-2 i \pi(\langle s, x\rangle+\langle t, y\rangle)} d s d t \\
& =A(u, v)(-y, x) \overline{A(v, w)(-y, x)} .
\end{aligned}
$$


40 A. Bonami, B. Demange and P. Jaming

\section{Heisenberg inequality for the ambiguity function.}

We show here that the ambiguity function is subject to sharp inequalities of Heisenberg type. We give first a directional version of Heisenberg's inequality (1.1) in the context of ambiguity functions, with an elementary proof.

Theorem 5.1 For $u, v \in L^{2}\left(\mathbb{R}^{d}\right)$, for every $i=1, \ldots, d$ and every $a, b \in \mathbb{R}$, one has the following inequality:

$$
\begin{gathered}
\iint_{\mathbb{R}^{2 d}}\left|x_{i}-a\right|^{2}|A(u, v)(x, y)|^{2} d x d y \iint_{\mathbb{R}^{2 d}}\left|y_{i}-b\right|^{2}|A(u, v)(x, y)|^{2} d x d y \\
\geq \frac{\|u\|_{L^{2}\left(\mathbb{R}^{d}\right)}^{4}\|v\|_{L^{2}\left(\mathbb{R}^{d}\right)}^{4}}{4 \pi^{2}}
\end{gathered}
$$

Moreover equality holds in (5.1), with $u$ and $v$ non identically 0 , if and only if there exists $\mu, \nu \in L^{2}\left(\mathbb{R}^{d-1}\right), \alpha>0$ and $\beta, \gamma \in \mathbb{R}$ such that

$$
\begin{aligned}
& u(t)=\mu\left(t_{1}, \ldots, t_{i-1}, t_{i+1}, \ldots, t_{d}\right) e^{2 i \pi \beta t_{i}} e^{-\alpha / 2\left|t_{i}-\gamma\right|^{2}} \\
& v(t)=\nu\left(t_{1}, \ldots, t_{i-1}, t_{i+1}, \ldots, t_{d}\right) e^{2 i \pi(b+\beta) t_{i}} e^{-\alpha / 2\left|t_{i}-a-\gamma\right|^{2}}
\end{aligned}
$$

To prove the theorem, we will need the following lemma that has its own interest :

Lemma 5.2 Let $u, v \in L^{2}\left(\mathbb{R}^{d}\right)$ be both non identically zero and let $i=$ $1, \ldots, d$. The following are equivalent:

(a) $\iint\left|x_{i}\right|^{2}|A(u, v)(x, y)|^{2} d x d y<+\infty$.

(b) For all $a \in \mathbb{R}, \iint\left|x_{i}-a\right|^{2}|A(u, v)(x, y)|^{2} d x d y<+\infty$.

(c) $\int\left|t_{i}\right|^{2}|u(t)|^{2} d t<+\infty$ and $\int\left|t_{i}\right|^{2}|v(t)|^{2} d t<+\infty$.

(d) For all $a, b \in \mathbb{R}$,

$$
\int\left|t_{i}-a\right|^{2}|u(t)|^{2} d t<+\infty \text { and } \int\left|t_{i}-b\right|^{2}|v(t)|^{2} d t<+\infty
$$

Remark: Note that, if $u \in L^{2}\left(\mathbb{R}^{d}\right)$ and if $\int\left|t_{i}\right|^{2}|u(t)|^{2} d t<+\infty$, then $t_{i}|u(t)|^{2} \in L^{1}\left(\mathbb{R}^{d}\right)$. 
Proof. Let us first remark that $(a) \Longleftrightarrow(b)$ and $(c) \Longleftrightarrow(d)$. Indeed, it is sufficient to use the triangle inequality

$$
|x|^{2} \leq 2\left(|x-a|^{2}+|a|^{2}\right)
$$

Moreover, $(b)$ and $(d)$ may be replaced by $\left(b^{\prime}\right)$ and $\left(d^{\prime}\right)$, where "for all" has been changed into "for some". Let us prove that $(a)$ implies $\left(d^{\prime}\right)$. With the notations of Lemma 4.2, Parseval identity gives:

$$
\begin{aligned}
\iint\left|x_{i}\right|^{2}|A(u, v)(x, y)|^{2} d x d y & =\int\left|x_{i}\right|^{2} \int\left|\widehat{h_{x}}(y)\right|^{2} d y d x \\
& =\int\left|x_{i}\right|^{2} \int\left|h_{x}(t)\right|^{2} d t d x \\
& =\iint\left|x_{i}\right|^{2}\left|u\left(t+\frac{x}{2}\right) \overline{v\left(t-\frac{x}{2}\right)}\right|^{2} d t d x \\
& =\int\left(\int\left|\eta_{i}-\xi_{i}\right|^{2}|v(\xi)|^{2} d \xi\right)|u(\eta)|^{2} d \eta
\end{aligned}
$$

So, if (a) holds, for almost every $\eta$,

$$
|u(\eta)|^{2} \int\left|\xi_{i}-\zeta_{i}\right|^{2}|v(\xi)|^{2} d \xi<+\infty
$$

As we assumed that $u \neq 0$, there exists $\eta$ such that $u(\eta) \neq 0$, and the first inequality in $\left(d^{\prime}\right)$ holds with $a=\eta$. Since $u$ and $v$ play the same role, we conclude for the second part similarly.

Conversely, if $(c)$ holds, the right hand side of (5.2) is finite, and $(a)$ holds also.

Proof of Theorem 5.1. Let us start by proving the inequality. Set $A=$ $A(u, v)$. We may assume that

$$
\left[\iint\left|x_{i}\right|^{2}|A(x, y)|^{2} d x d y\right]\left[\iint\left|y_{i}\right|^{2}|A(x, y)|^{2} d x d y\right]<+\infty
$$

so that both factors are finite, and, by homogeneity, that

$$
\|u\|_{L^{2}\left(\mathbb{R}^{d}\right)}=\|v\|_{L^{2}\left(\mathbb{R}^{d}\right)}=1 .
$$

Moreover, replacing $u$ and $v$ by translates of these functions (with the same translation), we may assume that

$$
-\int t_{i}|u(t)|^{2} d t=\int t_{i}|v(t)|^{2} d t=\frac{\bar{a}}{2} .
$$


Heisenberg's inequality (1.1) applied to $h_{x}(t)=u\left(t+\frac{x}{2}\right) \overline{v\left(t-\frac{x}{2}\right)}$ implies that, for any $b \in \mathbb{R}^{d}$ :

$$
\frac{1}{4 \pi} \int\left|h_{x}(t)\right|^{2} d t \leq\left(\int\left|t_{i}\right|^{2}\left|h_{x}(t)\right|^{2} d t\right)^{1 / 2}\left(\int\left|y_{i}-b\right|^{2}|A(u, v)(x, y)|^{2} d y\right)^{1 / 2} \text {. }
$$

Integrating this inequality with respect to the $x$-variable and appealing to Cauchy-Schwarz inequality, we get:

$$
\frac{1}{4 \pi}=\frac{\|u\|^{2}\|v\|^{2}}{4 \pi} \leq\left(\iint\left|t_{i}\right|^{2}\left|h_{x}(t)\right|^{2} d t d x\right)^{\frac{1}{2}}\left(\iint\left|y_{i}-b\right|^{2}|A(u, v)(x, y)|^{2} d x d y\right)^{\frac{1}{2}} .
$$

Let us now transform the first factor on the right hand side of this expression. We write

$$
\left|t_{i}\right|^{2}=\left(t_{i}+\frac{x_{i}-a}{2}\right)\left(t_{i}-\frac{x_{i}-a}{2}\right)+\frac{\left|x_{i}-a\right|^{2}}{4} .
$$

The second term which appears is, by Parseval identity, equal to

$$
\frac{1}{4} \iint\left|x_{i}-a\right|^{2}|A(x, y)|^{2} d x d y \text {. }
$$

The first term is equal to

$$
\iint\left(\eta_{i}-\frac{a}{2}\right)\left(\xi_{i}+\frac{a}{2}\right)|u(\eta)|^{2}|v(\xi)|^{2} d \eta d \xi=-\frac{1}{4}|\bar{a}-a|^{2} \leq 0
$$

using (5.3). Finally, including these results in (5.5), we obtain the desired inequality.

Assume now that we have equality. Let us remark that, using properties $(a)$ and (b) of Lemma 4.1, we may as well assume that the constants $a$ and $b$ are 0 . Moreover, up to a same translations in space and frequency, we may again assume that $-\int t_{i}|u(t)|^{2} d t=\int t_{i}|v(t)|^{2} d t=\frac{\bar{a}}{2}$, and $-\int t_{i}|\mathcal{F} u(t)|^{2} d t=$ $\int t_{i}|\mathcal{F} v(t)|^{2} d t=\frac{\bar{b}}{2}$. Let $h_{x}(t)=u\left(t+\frac{x}{2}\right) \overline{v\left(t-\frac{x}{2}\right)}$ as before. Then, to have equality in (5.1), we have equality in (5.6), i.e. we have $\bar{a}=a=0$. Similarly, exchanging the roles of the $x$ and $y$ variables, we also have $\bar{b}=b=0$.

We then have equality in Cauchy-Schwarz inequality (5.5). This implies that

$$
x \mapsto \int\left|t_{i}\right|^{2}\left|h_{x}(t)\right|^{2} d t \text { and } x \mapsto \int\left|y_{i}\right|^{2}\left|\widehat{h_{x}}(y)\right|^{2} d y
$$

are proportional. 
Further, for almost every $x$, we also have equality in Heisenberg's inequality (5.4). From now on, we assume for simplicity that $i=1$. We then get that, for almost every $x, h_{x}$ is a Gaussian in the $t_{1}$ variable:

$$
h_{x}(t)=C\left(x, t^{\prime}\right) e^{-\pi \alpha(x)\left|t_{1}\right|^{2}} .
$$

where $t^{\prime}=\left(t_{2}, \ldots, t_{d}\right) \in \mathbb{R}^{d-1}, C\left(x, t^{\prime}\right)$ is not identically 0 and $\alpha(x)>0$ for those $x$ for which $C\left(x, t^{\prime}\right) \neq 0$.

Let us first prove that $\alpha$ does not depend on $x$. With this expression of $h_{x}$, we get that

$$
\int\left|t_{1}\right|^{2}\left|h_{x}(t)\right|^{2} d t=\|C(x, .)\|_{L^{2}\left(\mathbb{R}^{d-1}\right)}^{2} \frac{\sqrt{\pi}}{2}\left(\frac{1}{2 \pi \alpha(x)}\right)^{3 / 2}
$$

whereas (with Parseval identity)

$$
\int\left|y_{i}\right|^{2}\left|\widehat{h_{x}}(y)\right|^{2} d y=\|C(x, .)\|_{L^{2}\left(\mathbb{R}^{d-1}\right)}^{2} \frac{1}{4 \pi} \sqrt{\frac{\alpha(x)}{2}} .
$$

But these two functions are proportional only if $\alpha(x)$ is a constant, say $\alpha(x)=\alpha$.

Taking Fourier transforms, we get that

$$
A(u, v)(x, y)=\frac{1}{\sqrt{\alpha}} \widehat{C}\left(x,-y^{\prime}\right) e^{-\frac{\pi}{\alpha}\left|y_{1}\right|^{2}}
$$

where $\widehat{C}(x,$.$) is the Fourier transform in \mathbb{R}^{d-1}$ of $C(x,$.$) . In particular, as$ $A(u, v)$ is continuous, $\widehat{C}$ is also continuous.

Further, if one has equality in (5.1) for $u, v$, one has again this equality if $u, v$ are replaced by their Fourier transform, as $A(\widehat{u}, \widehat{v})(x, y)=A(u, v)(-y, x)$. So, we have

$$
A(u, v)(x, y)=D\left(y, x^{\prime}\right) e^{-\frac{\pi}{\beta}\left|x_{1}\right|^{2}}
$$

for some function $D$ and some $\beta>0$. Comparing (5.7) and (5.8), we get that

$$
A(u, v)(x, y)=E\left(x^{\prime}, y^{\prime}\right) e^{-\frac{\pi}{\beta}\left|x_{1}\right|^{2}} e^{-\frac{\pi}{\alpha}\left|y_{1}\right|^{2}}
$$

for some function $E \in L^{2}\left(\mathbb{R}^{d-1} \times \mathbb{R}^{d-1}\right)$. Taking the inverse Fourier transform in the $y$ variable, we get

$$
u\left(t+\frac{x}{2}\right) \overline{v\left(t-\frac{x}{2}\right)}=\widetilde{E}\left(x^{\prime}, t^{\prime}\right) e^{-\frac{\pi}{\beta}\left|x_{1}\right|^{2}} e^{-\pi \alpha\left|t_{1}\right|^{2}} .
$$


So, setting again $\eta=t+\frac{x}{2}, \xi=t-\frac{x}{2}$, we get

$$
u(\eta) \overline{v(\xi)}=\widetilde{E}\left(\eta^{\prime}-\xi^{\prime}, \frac{\eta^{\prime}+\xi^{\prime}}{2}\right) e^{-\frac{\pi}{\beta}\left(\eta_{1}-\xi_{1}\right)^{2}} e^{-\frac{\pi \alpha}{4}\left(\eta_{1}+\xi_{1}\right)^{2}}
$$

This is only possible if $\beta=\frac{4}{\alpha}$ and $u(x)=\mu\left(\widehat{x}_{i}\right) e^{-\frac{\pi \alpha}{2}\left|x_{i}\right|^{2}}, v(x)=\nu\left(\widehat{x}_{i}\right) e^{-\frac{\pi \alpha}{2}\left|x_{i}\right|^{2}}$ with $\mu, \nu \in L^{2}\left(\mathbb{R}^{d-1}\right)$.

We now translate the last theorem in terms of inequalities on covariance matrices, as it is classical for inequality (1.1) (see for instance [9]).

Proof of Theorem 1.5. First, it follows from Lemma 5.2 that $X$ and $Y$ have moments of order 2. Let us prove that $X$ and $Y$ are not correlated. Without loss of generality, we may assume that

$$
\int t_{i}|u(t)|^{2} d t=\int t_{i}|v(t)|^{2} d t=\int t_{i}|\mathcal{F} u(t)|^{2} d t=\int t_{i}|\mathcal{F} v(t)|^{2} d t=0
$$

for all $i$, so that $X$ and $Y$ are centered. Let us show that

$$
\iint x_{i} y_{j}|A(u, v)|^{2} d x d y=0
$$

Using Plancherel identity, we are led to consider

$$
\iint x_{i}\left[\partial_{t_{j}} h_{x}(t) \overline{h_{x}(t)}+h_{x}(t) \overline{\partial_{t_{j}} h_{x}(t)}\right] d x d t
$$

Writing $x_{i}=t+x_{i} / 2-\left(t-x_{i} / 2\right)$, and

$$
\partial_{t_{j}} h_{x}(t)=u\left(t+\frac{x}{2}\right) \overline{\partial_{t_{j}} v\left(t-\frac{x}{2}\right)}+\partial_{t_{j}} u\left(t+\frac{x}{2}\right) \overline{v\left(t-\frac{x}{2}\right)}
$$

we get eight terms. For four of them we get directly 0 . The sum of the last four may be written, after changes of variables, as

$$
\int_{\mathbb{R}^{d}} t_{i} \partial_{t_{j}}\left[|u(t)|^{2}\right] d t \times \int_{\mathbb{R}^{d}}|v(t)|^{2} d t-\int_{\mathbb{R}^{d}}|u(t)|^{2} d t \times \int_{\mathbb{R}^{d}} t_{i} \partial_{t_{j}}\left[|v(t)|^{2}\right] d t .
$$

After an integration by parts, the two terms give $\delta_{i j}$, so that their difference is 0 .

Let us now prove the second assertion. Let $C$ be an automorphism of $\mathbb{R}^{d}$. For a function $f \in L^{2}\left(\mathbb{R}^{d}\right)$, we consider $f_{C}$ the function given by $f_{C}(t)=|\operatorname{det}(C)|^{-1 / 2} f\left(C^{-1} t\right)$. Then a simple change of variables shows that the probability density of $\left(C X,{ }^{t} C^{-1} Y\right)$ is $\left|A\left(u_{C}, v_{C}\right)\right|^{2}$. Eventually changing $u$ and $v$ into $u_{C}$ and $v_{C}$ we may assume that $V(Y)$ is the identity matrix. 
Moreover, we may also assume that $V(X)$ is diagonal. Then the inequality follows from Theorem 5.1. Equality holds only if all eigenvalues are equal to $4 \pi^{2}$, which means that $u$ and $v$ are Gaussian functions.

From Theorem 1.5, we immediately obtain that

$$
\operatorname{det}(V(X)) \times \operatorname{det}(V(Y)) \geq\left(4 \pi^{2}\right)^{-2 d} .
$$

Equality holds only for Gaussian functions. Another (much less elementary) proof of (5.9) can be obtained using the entropy inequality of Lieb and the theorem of Shannon (see [11], Section 6).

The same inequality holds for traces instead of determinants. We state it independently.

Corollary 5.3 Let $u, v \in L^{2}\left(\mathbb{R}^{d}\right)$ be both non identically zero and $a, b \in \mathbb{R}^{d}$. Then

$$
\begin{aligned}
\iint_{\mathbb{R}^{2 d}}\|x-a\|^{2}|A(u, v)(x, y)|^{2} d x d y & \times \iint_{\mathbb{R}^{2 d}}\|y-b\|^{2}|A(u, v)(x, y)|^{2} d x d y \\
& \geq \frac{d^{2}\|u\|_{L^{2}\left(\mathbb{R}^{d}\right)}^{4}\|v\|_{L^{2}\left(\mathbb{R}^{d}\right)}^{4}}{4 \pi^{2}}
\end{aligned}
$$

with equality if and only if there exists $\lambda, \nu \in \mathbb{C}^{*}, \alpha>0$ and $\beta, \gamma \in \mathbb{R}^{d}$ such that

$$
u(t)=\lambda e^{2 i \pi\langle\beta, t\rangle} e^{-\alpha / 2\|t-\gamma\|^{2}} \quad \text { and } \quad v(t)=\nu e^{2 i \pi\langle-b+\beta, t\rangle} e^{-\alpha / 2\|t+a-\gamma\|^{2}} .
$$

\section{Uncertainty principles for the ambiguity function.}

We first prove the following uncertainty principle for the ambiguity function, which also gives a characterization of Hermite functions:

Theorem 6.1 Let $u, v \in L^{2}\left(\mathbb{R}^{d}\right)$ be non identically vanishing. If

$$
\begin{aligned}
& \iint_{\mathbb{R}^{2 d}}|A(u, v)|^{2} \frac{e^{\pi\|x\|^{2}}}{(1+\|x\|)^{N}} d x d y<+\infty \quad \text { and } \\
& \iint_{\mathbb{R}^{2 d}}|A(u, v)|^{2} \frac{e^{\pi\|y\|^{2}}}{(1+\|y\|)^{N}} d x d y<+\infty
\end{aligned}
$$

for all $j=1, \cdots, d$, then there exists $a, w \in \mathbb{R}^{d}$ such that both $u$ and $v$ are of the form

$$
P(x) e^{2 i \pi\langle w, x\rangle} e^{-\pi\|x-a\|^{2}}
$$

with $P$ a polynomial. 
Let us first remark that when $u=v$, since the Fourier transform of $|A(u, u)|^{2}$ taken at $(x, y)$ is equal to $|A(u, u)|^{2}(-y, x)$, the result follows immediately from Theorem 1.3. Our aim is to prove that it is also valid in the general case. We first prove a weaker statement.

Proposition 6.2 Let $u, v \in L^{2}\left(\mathbb{R}^{d}\right)$ be non identically vanishing. If

$$
\iint_{\mathbb{R}^{d} \times \mathbb{R}^{d}} \frac{|A(u, v)(x, y)|^{2}}{(1+\|x\|+\|y\|)^{N}} e^{\pi\left(\|x\|^{2}+\|y\|^{2}\right)} d x d y<+\infty
$$

then there exists $a, w \in \mathbb{R}^{d}$ such that both $u$ and $v$ are of the form

$$
P(x) e^{2 i \pi\langle w, x\rangle} e^{-\pi\|x-a\|^{2}}
$$

with $P$ a polynomial.

Before starting the proof of Proposition 6.2, let us state two lemmas. The first one is elementary and well known.

Lemma 6.3 Let $u, v \in L^{2}\left(\mathbb{R}^{d}\right)$ be non identically vanishing. Then

$$
u(x)=P(x) e^{2 i \pi\langle\alpha, x\rangle} e^{-\pi\|x-a\|^{2}} \text { and } v(x)=Q(x) e^{2 i \pi\langle\alpha, x\rangle} e^{-\pi\|x-a\|^{2}}
$$

with $P, Q$ polynomials and $a, \alpha \in \mathbb{R}^{d}$ if and only if there is a polynomial $R$ such that

$$
A(u, v)(x, y)=R(x, y) e^{2 i \pi(\langle\alpha, x\rangle+\langle a, y\rangle)} e^{-\frac{\pi}{2}\left(\|x\|^{2}+\|y\|^{2}\right)} .
$$

Moreover, $\operatorname{deg}(R)=\operatorname{deg}(P)+\operatorname{deg}(Q)$.

Lemma 6.4 Assume that $u, v \in L^{2}\left(\mathbb{R}^{d}\right)$, with $u(x)=P(x) e^{2 i \pi\langle a, x\rangle} e^{-\pi\|x\|^{2}}$, where $a \in \mathbb{C}^{d}$. Then the function $A(u, v)$ can be extended to an entire function on $\mathbb{C}^{2 d}$.

Proof. For $z, \zeta \in \mathbb{C}^{d}$, we note $\langle z, \zeta\rangle=\sum z_{i} \zeta_{i}$. Then

$$
A(u, v)(x, y)=e^{i \pi\langle x,(y+2 a)\rangle} \times \int P(t+x) \overline{v(t)} e^{-\pi\|t+x\|^{2}} e^{2 i \pi\langle t,(a+y)\rangle} d t
$$

This clearly makes sense for $x, y \in \mathbb{C}^{d}$, and defines an entire function. 
Proof of Proposition 6.2. By homogeneity, we may assume that $\|u\|_{L^{2}}=$ $\|v\|_{L^{2}}=1$. For each $w \in L^{2}\left(\mathbb{R}^{d}\right)$, we consider the function

$$
g_{w}=A(u, v) \overline{A(v, w)} .
$$

By Lemma 4.3, the Fourier transform of $g_{w}$ is given by $\mathcal{F} g_{w}(x, y)=g_{w}(-y, x)$. First step. There exists a polynomial $P$ such that

$$
A(u, v)(x, y) \overline{A(v, u)}(x, y)=P(x, y) e^{-\pi\left(\|x\|^{2}+\|y\|^{2}\right)} .
$$

Proof. We consider here the function $g_{u}$. As $g_{u}$ is (up to a change of variable) its own Fourier transform, by Proposition 3.2, it is sufficient to prove that

$$
\int\left|g_{u}(x, y)\right| \frac{e^{\pi\left(\|x\|^{2}+\|y\|^{2}\right)}}{(1+\|x\|+\|y\|)^{N}} d x d y<\infty .
$$

It follows immediately from the assumption on $A(u, v)$, using Cauchy-Schwarz inequality and the fact that $\overline{A(v, u)(x, y)}=A(u, v)(-x,-y)$.

To complete the proof of the proposition, it is sufficient to prove that $A(u, v)$ extends to an entire function of order 2. Indeed, Lemma 2.3 then implies that

$$
A(u, v)(x, y)=R(x, y) e^{Q(x, y)},
$$

where $R$ is a polynomial and $Q$ a polynomial of degree at most 2. But, as

$$
\begin{aligned}
A(u, v)(x, y) A(u, v)(-x,-y) & =A(u, v)(x, y) \overline{A(v, u)(x, y)} \\
& =P(x, y) e^{-\pi\left(\|x\|^{2}+\|y\|^{2}\right)},
\end{aligned}
$$

we get $Q(x, y)=\langle\beta, x\rangle+\langle\gamma, y\rangle-\frac{\pi}{2}\left(\|x\|^{2}+\|y\|^{2}\right)$ for some constants $\beta, \gamma \in \mathbb{C}^{d}$. Next, Condition (6.2) implies that $\beta, \gamma$ are purely imaginary, $\beta=2 i \pi \alpha, \gamma=$ $2 i \pi a$ with $a, \alpha \in \mathbb{R}^{d}$ so that

$$
A(u, v)(x, y)=R(x, y) e^{2 i \pi(\langle\alpha, x\rangle+\langle a, y\rangle)} e^{-\frac{\pi}{2}\left(\|x\|^{2}+\|y\|^{2}\right)},
$$

with $R$ a polynomial. Lemma 6.3 allows to conclude. So, we have finished the proof once we have proved the second step.

Second step. The function $A(u, v)$ extends to an entire function of order 2 .

Proof. To prove this, we first show that, for each $w \in L^{2}\left(\mathbb{R}^{d}\right)$, the function $g_{w}$ extends to an entire function of order 2. Since, up to a change of variable, $g_{w}$ coincides with its Fourier transform, it is sufficient to show that

$$
\int\left|g_{w}(x, y)\right| e^{\frac{\pi}{4}\left(\|x\|^{2}+\|y\|^{2}\right)} d x d y<\infty .
$$


This last inequality follows from the fact that $\left|g_{w}\right| \leq|A(u, v)|\|w\|_{L^{2}}$, and from the assumption on $A(u, v)$. We get the estimate

$$
\left|g_{w}(z, \zeta)\right| \leq C\|w\| e^{4 \pi\left(\|z\|^{2}+\|\zeta\|^{2}\right)}
$$

for all $(z, \zeta) \in \mathbb{C}^{d} \times \mathbb{C}^{d}$, with a constant $C$ which does not depend on $w$. In order to conclude this step, it is sufficient to show that there exists a constant $C$ such that, for each $(z, \zeta) \in \mathbb{C}^{d} \times \mathbb{C}^{d}$, we may find $w_{z, \zeta}$ which is of the form required in Lemma 6.4 , such that

$$
\left|A\left(w_{z, \zeta}, v\right)(z, \zeta)\right| \geq C^{-1} e^{-C\left(\|z\|^{2}+\|\zeta\|^{2}\right)},
$$

and

$$
\left\|w_{z, \zeta}\right\| \leq C^{-1} e^{C\left(\|z\|^{2}+\|\zeta\|^{2}\right)} .
$$

By density of the Hermite functions we can choose a polynomial $P_{0}$ such that

$$
\int P_{0}(t) \overline{v(t)} e^{-\pi\|t\|^{2}} d t=1 .
$$

We then define $w_{z, \zeta}$ by

$$
w_{z, \zeta}(t)=P_{0}(t-z) e^{2 \pi\langle z-i \zeta, t\rangle} e^{-\pi\|t\|^{2}} .
$$

It follows from the choice of $P_{0}$ that

$$
A\left(w_{z, \zeta}, v\right)(z, \zeta)=e^{\pi(\langle z, z\rangle-i\langle z, \zeta\rangle)} .
$$

Then (6.7) and (6.8) follow from direct computations. Finally, since

$$
A(u, v) \overline{A\left(v, w_{z, \zeta}\right)}
$$

extends to an entire function for each $z, \zeta$, and since the second factor is also entire and does not vanish in a neighborhood of $(-z,-\zeta) \in \mathbb{C}^{d}, A(u, v)$ extends also to an entire function. The fact that it is of order 2 follows from (6.6), (6.7) and (6.8).

We have completed the proof of Proposition 6.2.

Proof of Theorem 6.1. With the weaker assumption (6.1), we conclude that (6.4) also holds, using the directional theorem for Fourier transforms. We claim that $A(u, v)$ is an analytic function of each of the variables $x$ and $y$. Indeed, as before, for every function $w \in L^{2}\left(\mathbb{R}^{d}\right)$, the product $A(u, v) \overline{A(v, w)}$ extends to a holomorphic function of $x, y$ being fixed, as well as to a holomorphic function of $y, x$ being fixed. When choosing $w$ as 
before, we conclude that the function extends to an entire function of order 2 in $x$, for fixed $y$. So, for almost every fixed $y, A(u, v)(x, y)$ may be written as $R_{y}(x) e^{2 i \pi\langle\alpha(y), x\rangle} e^{-\frac{\pi}{2}\|x\|^{2}}$, with $R_{y}$ a polynomial of degree at most $N$. It follows that the continuous function

$$
e^{\pi\left(\|x\|^{2}+\|y\|^{2}\right)}|A(u, v)(x, y)|^{2}
$$

is a polynomial of degree at most $2 N$ in each variable $x, y$. So it is a polynomial, and the assumption (6.2) is satisfied. We can now use Proposition 6.2 to conclude.

In particular, it follows from Theorem 6.1 that there does not exist two non zero functions $u, v$ which satisfy Condition (6.1) for $N \leq d$. In this case, where the conclusion is that $u$ or $v$ is identically 0 , the condition can be relaxed into a directional condition as for the case of the Fourier transform.

Corollary 6.5 Assume that

$$
\begin{aligned}
& \iint_{\mathbb{R}^{2 d}}|A(u, v)|^{2} \frac{e^{\pi\left|x_{j}\right|^{2}}}{\left(1+\left|x_{j}\right|\right)^{M}} d x d y<+\infty \quad \text { and } \\
& \iint_{\mathbb{R}^{2 d}}|A(u, v)|^{2} \frac{e^{\pi\left|y_{j}\right|^{2}}}{\left(1+\left|y_{j}\right|\right)^{N}} d x d y<+\infty
\end{aligned}
$$

for some $j=1, \ldots$, d. If $\min \{M, N\}=1$, then $u$ or $v$ vanishes.

Proof. When $d=1$, the result follows from Theorem 6.1. Let us now consider the case $d>1$. We assume that the condition is satisfied for $j=1$. For $t^{\prime} \in \mathbb{R}^{d-1}$ and for $f$ in $L^{2}\left(\mathbb{R}^{d}\right)$, we define $f_{t^{\prime}}\left(t_{1}\right)=f\left(t_{1}, t^{\prime}\right)$. It follows from Plancherel identity that

$$
\int_{\mathbb{R}^{d-1}}|A(u, v)(x, y)|^{2} d y^{\prime}=\int_{\mathbb{R}^{d-1}}\left|A\left(u_{t^{\prime}-\frac{x^{\prime}}{2}}, v_{t^{\prime}+\frac{x^{\prime}}{2}}\right)\left(x_{1}, y_{1}\right)\right|^{2} d y^{\prime} .
$$

Changing variables, and using Fubini's Theorem, it follows that, for almost every $\xi^{\prime}$ and $\eta^{\prime}$ in $\mathbb{R}^{d-1}$,

$$
\iint_{\mathbb{R} \times \mathbb{R}} \frac{\left|A\left(u_{\eta^{\prime}}, v_{\xi^{\prime}}\right)\left(x_{1}, y_{1}\right)\right|^{2}}{\left(1+\left|x_{1}\right|+\left|y_{1}\right|\right)^{2}} e^{\pi\left(\left|x_{1}\right|^{2}+\left|y_{1}\right|^{2}\right)} d x_{1} d y_{1}<+\infty .
$$

It follows from the one dimensional case that either $u_{\eta^{\prime}}$ or $v_{\xi^{\prime}}$ is identically 0 . So, for almost every $\xi^{\prime}$ and $\eta^{\prime}$ in $\mathbb{R}^{d-1}, A\left(u_{\eta^{\prime}}, v_{\xi^{\prime}}\right)=0$. It follows that $A(u, v)=0$.

As in section 3, we may deduce from Theorem 6.1 a version of Hardy's theorem for the ambiguity function. Let us remark that the constraints on degrees are always elementary. The case when $N=0$ has also been considered in $([15])$. 
50 A. Bonami, B. Demange and P. Jaming

Corollary 6.6 Let $u, v \in L^{2}\left(\mathbb{R}^{d}\right)$ and assume that

$$
\begin{aligned}
|A(u, v)(x, y)| & \leq K(1+\|x\|)^{N} e^{-\frac{\pi}{2}\langle B x, x\rangle} \\
\text { and } \quad|A(u, v)(x, y)| & \leq K(1+\|y\|)^{N} e^{-\frac{\pi}{2}\langle C y, y\rangle} .
\end{aligned}
$$

We have the following implications.

1. If $B-C^{-1}$ is positive, non zero, then either $u$ or $v=0$.

2. If $B=C^{-1}$, there are polynomials $P, Q$ of degree $\leq N$ and constants $\omega, a \in \mathbb{R}^{d}$ such that

$$
\begin{aligned}
u(x) & =P(x) e^{2 i \pi\langle\omega, x\rangle} e^{-\pi\langle B(x-a), x-a\rangle} \\
\text { and } \quad v(x) & =Q(x) e^{2 i \pi\langle\omega, x\rangle} e^{-\pi\langle C(x-a), x-a\rangle} .
\end{aligned}
$$

Remark: This corollary implies in particular that if $u_{0}(x)=P_{0}(x) e^{-\alpha\|x\|^{2}}$, $v_{0}(x)=Q_{0}(x) e^{-\alpha\|x\|^{2}}$ with $P_{0}, Q_{0}$ polynomials and if $|A(u, v)|=\left|A\left(u_{0}, v_{0}\right)\right|$ then $u, v$ are of the form

$$
u(x)=P(x) e^{2 i \pi\langle\omega, x\rangle-\alpha\|x-a\|^{2}} \quad v(x)=Q(x) e^{2 i \pi\langle\omega, x\rangle-\alpha\|x-a\|^{2}}
$$

with $P, Q$ polynomials and $\omega, a \in \mathbb{R}^{d}$.

The problem of finding $u, v$ from $u_{0}, v_{0}$ is known as the radar ambiguity problem and has been considered by Bueckner [5] and de Buda [4] for $u_{0}, v_{0}$ as above. This remark corrects the proof of [4].

Further references on this problem may be found in [20] and [12].

Let us finally give a Morgan type theorem.

Theorem 6.7 Let $1<p<2$, and let $q$ be the conjugate exponent. Assume that $u, v \in L^{2}\left(\mathbb{R}^{d}\right)$ satisfy

$$
\begin{array}{r}
\iint_{\mathbb{R}^{d} \times \mathbb{R}^{d}}|A(u, v)(x, y)|^{2} e^{\frac{2 \pi}{p} a^{p}\left|x_{j}\right|^{p}} d x d y<+\infty \\
\text { and } \iint_{\mathbb{R}^{d} \times \mathbb{R}^{d}}|A(u, v)(x, y)|^{2} e^{\frac{2 \pi}{q} b^{q}\left|y_{j}\right|^{q}} d x d y<+\infty
\end{array}
$$

for some $j=1, \ldots, d$ and for some positive constants $a$ and $b$. Then either $u$ or $v$ vanish if $a b>\left|\cos \left(\frac{p \pi}{2}\right)\right|^{\frac{1}{p}}$. If $a b<\left|\cos \left(\frac{p \pi}{2}\right)\right|^{\frac{1}{p}}$, there exists a non zero function $u$ such that the two conditions are satisfied by $A(u, u)$. 
Proof. It is sufficient to consider the one dimensional case. Otherwise, the proof works as in Corollary 6.5. Let us prove the first assertion. If we proceed as in the proof of Theorem 6.1 , we conclude at once that

$$
A(u, v)(x, y) A(u, v)(-x,-y)=0
$$

using the similar result on Fourier transforms. It remains to show that $A(u, v)$ is an analytic function of each variable, which is obtained in the same way as before using an auxiliary function $w$.

Let us now prove that, for $a b<\left|\cos \left(\frac{p \pi}{2}\right)\right|^{\frac{1}{p}}$, there exists a non zero function $u$ such that the two conditions are satisfied by $A(u, u)$. Using Plancherel formula, the first condition may as well be written as

$$
\iint_{\mathbb{R} \times \mathbb{R}}\left|u\left(t-\frac{x}{2}\right)\right|^{2}\left|u\left(t+\frac{x}{2}\right)\right|^{2} e^{\frac{2 \pi}{p} a^{p}|x|^{p}} d x d t<+\infty .
$$

Using the same change of variable as before, and the inequality $|\eta-\xi| \leq$ $2^{p-1}\left(|\eta|^{p}+|\xi|^{p}\right)$, we see that this integral is bounded by the square of the integral

$$
\int_{\mathbb{R}}|u(\xi)|^{2} e^{\frac{4 \pi}{p}\left(2^{1-2 / p} a\right)^{p}|\xi|^{p}} d \xi .
$$

For the second integral, we use Lemma 4.1 to write it in terms of the Fourier transform of $u$. We obtain that it is bounded by

$$
\int_{\mathbb{R}}|\widehat{u}(\xi)|^{2} e^{\frac{4 \pi}{q}\left(2^{1-2 / q} b\right)^{q}|\xi|^{q}} d \xi
$$

The fact that there is a non zero function $u$ for which both integrals are finite is an easy consequence of Theorem 1.4 and Schwarz inequality, since $\left(2^{1-2 / p} a\right) \times\left(2^{1-2 / q} b\right)<\left|\cos \left(\frac{p \pi}{2}\right)\right|^{\frac{1}{p}}$.

Remark: At this stage, we would like to point out that we have not been able to replace the conditions of Theorem 6.1 by the condition

$$
\begin{aligned}
& \iint \frac{|A(u, v)(x, y)|^{2}}{(1+\|x\|+\|y\|)^{N}} e^{2 \pi|\langle x, y\rangle|} d x d y<+\infty \\
\text { or at least } & \iint \frac{|A(u, v)(x, y)|^{2}}{(1+\|x\|+\|y\|)^{N}} e^{2 \pi\|x\|\|y\|} d x d y<+\infty .
\end{aligned}
$$

Also, we do not know whether weaker conditions, with $|A(u, v)(x, y)|$ in place of its square, are sufficient. 
52 A. Bonami, B. Demange and P. Jaming

\section{Appendix}

We give here a simplified proof of Beurling-Hörmander's Theorem, which may be useful for elementary courses on Fourier analysis. All ideas are contained in Section 2.

We want to prove that a function $f \in L^{1}(\mathbb{R})$ which satisfies the inequality

$$
\iint_{\mathbb{R} \times \mathbb{R}}|f(x)||\widehat{f}(\xi)| e^{2 \pi|x||\xi|} d x d \xi<+\infty
$$

is identically 0. It is sufficient to show that the function $g=e^{-\pi x^{2}} * f$ is identically 0. Indeed, the Fourier transform of $g$ is equal to $e^{-\pi \xi^{2}} \widehat{f}$. If it is 0 , then $f$ vanishes also. Now $g$ extends to an entire function of order 2 in the complex plane. We note also $g$ its extension. We claim that, moreover,

$$
\iint_{\mathbb{R} \times \mathbb{R}}|g(x)||\widehat{g}(\xi)| e^{2 \pi|x||\xi|} d x d \xi<+\infty .
$$

Indeed, replacing $g$ and $\widehat{g}$ by their values in terms of $f$ and $\widehat{f}$ and using Fubini's theorem, we are led to prove that the quantity

$$
\int_{\mathbb{R}} e^{-\pi\left((x-y)^{2}-2|x||\xi|+2|y||\xi|+\xi^{2}\right)} d x
$$

is bounded independently of $y$ and $\xi$. Taking $x-y$ as the variable, it is sufficient to prove that

$$
\int_{\mathbb{R}} e^{-\pi\left(x^{2}-2|x||\xi|+\xi^{2}\right)} d x
$$

is bounded by 2 , which follows from the fact that $x^{2}-2|x||\xi|+\xi^{2}$ is either $(x-\xi)^{2}$ or $(x+\xi)^{2}$.

Now, for all $z \in \mathbb{C}$, we have the elementary inequality

$$
|g(z)| \leq \int_{\mathbb{R}}|\widehat{g}(\xi)| e^{2 \pi|z||\xi|} d \xi
$$

so that there exists some constant $C$ such that

$$
\int_{-\infty}^{+\infty}|g(x)| \sup _{|z|=|x|}|g(z)| d x \leq C .
$$

We claim that the holomorphic function

$$
G(z)=\int_{0}^{z} g(u) g(i u) d u
$$


is bounded by $C$. Once we know this, the end of the proof is immediate: $G$ is constant by Liouville's Theorem; so $g(u) g(i u)$ is identically 0 , which implies that $g$ is identically 0 .

It is clear from (6.12) that $G$ is bounded by $C$ on the axes. Let us prove that it is bounded by $C$ for $z=r e^{i \theta}$ in the first quadrant. Assume that $\theta$ is in the interval $(0, \pi / 2)$. By continuity, it is sufficient to prove that

$$
G_{\alpha}(z)=\int_{0}^{z} g\left(e^{-i \alpha} u\right) g(i u) d u
$$

is bounded by $2 C$ for all $\alpha \in(0, \theta)$. But the function $G_{\alpha}$ is an entire function of order 2, which is bounded by $C$ on the $y$-axis and on the half-line $\rho e^{i \alpha}$. By Phagmèn-Lindelhöf principle, it is bounded by $2 C$ inside the angular sector, which gives the required bound for $\left|G_{\alpha}(z)\right|$. A similar proof gives the same bound in the other quadrants.

\section{References}

[1] Auslander, L. And Tolimieri, R.: Radar ambiguity functions and group theory. SIAM J. Math Anal. 16 (1985), 577-601.

[2] BAGCHI, S.C. And RAY, S.K.: Uncertainty principles like Hardy's theorem on some Lie groups. J. Austral. Math. Soc. Ser. A 65 (1999), 289-302.

[3] DE Bruijn, N.G.: A theory of generalized functions with applications to Wigner distribution and Weyl correspondence. Nieuw Arch. Wisk. (3) 21 (1973), 205-280.

[4] DE BudA, R.: Signals that can be calculated from their ambiguity function. IEEE Trans. Inform. Theory IT-16 (1970), 195-202.

[5] Bueckner, H.F.: Signals having the same ambiguity functions. Technical Report 67-C-456, General Electric, Research and Development Center, Schnectady, N.Y., 1967.

[6] Cohen, L.: Time-frequency distributions - a review. Proc. IEEE 77:7 (1989), 941-981.

[7] Cook, C.E. And Bernfeld, M.: Radar signals - an introduction to theory and application. Academic Press (1967).

[8] Cowling, M.G. And Price, J.F.: Generalizations of Heisenberg's inequality. In: Harmonic Analysis (eds. G. Mauceri, F. Ricci and G. Weiss), LNM, no. 992. pp. 443-449, Springer, Berlin, 1983.

[9] Dembo, A., Cover, T.M. and Thomas, J.A.: Information-theoretic inequalities. IEEE Trans. Inform. Theory 37:6 (1991), 1501-1518.

[10] Flandrin, P.: Separability, positivity, and minimum uncertainty in timefrequency energy distributions. J. Math. Phys. 39:8 (1998), 4016-4040. 
[11] Folland, G.B. And Sitaram, A.: The uncertainty principle - a mathematical survey. J. Fourier Anal. Appl. 3 (1997), 207-238.

[12] Garrigós, G., Jaming Ph. and Poly, J.-B.: Zéros de fonctions holomorphes et contre-exemples en théorie des radars. Rencontres d'Analyse Complexe, Poitiers, 2001.

[13] Gel'Fand, I.M. And Shilov, G.E.: Fourier transforms of rapidly increasing functions and questions of uniqueness of the solution of Cauchy's problem. Uspekhi Mat. Nauk 8:6 (1953), 3-54.

[14] Gel'Fand, I.M And Shilov, G.E.: Generalized functions, 2. Moscow, 1958 (Russian). English translation, Academic Press, 1968.

[15] Gröchenig, K. and Zimmermann, G.: Hardy's theorem and the shorttime Fourier transform of Schwartz functions. J. London Math. Soc. (2) 63:1 (2001), 93-103.

[16] Hardy, G. H.: A theorem concerning Fourier transforms. J. London Math. Soc. 8 (1933), 227-231.

[17] Havin, V. And JöRICKe, B.: The uncertainty principle in harmonic analysis. Springer-Verlag, Berlin, 1994.

[18] Hörmander, L.: A uniqueness theorem of Beurling for Fourier transform pairs. Ark. Mat. 29 (1991), 237-240.

[19] Jaming, Ph.: Principe d'incertitude qualitatif et reconstruction de phase pour la transformée de Wigner. C. R. Acad. Sci. Paris Sér. I Math. 327:3 (1998), 249-254.

[20] Jaming Ph.: Phase retrieval techniques for radar ambiguity functions. J. Fourier Anal. Appl. 5 (1999), 313-333.

[21] Janssen, A. J. E. M.: Optimality property of the gaussian window spectrogram. IEEE Trans. Inform. Theory 39:1 (1991), 202-204.

[22] Janssen, A. J. E. M.: Proof of a conjecture on the supports of Wigner distributions. J. Fourier Anal. Appl. 4:6 (1998), 723-726.

[23] Levin, B. JA.: Distribution of zeros of entire functions Transl. Math. Monogr. 5, AMS, Providence, 1980.

[24] Morgan, G. W.: A note on Fourier transforms. J. London Math. Soc. 9 (1934), 187-192.

[25] Narayanan, E.K. And Ray, S. K.: Beurling's theorem on $\mathbb{R}^{n}$. Preprint.

[26] NAZArov, F.: Local estimates for exponential polynomials and their applications to inequalities of the uncertainty principle type. Algebra i Analiz 5:4 (1993), 3-66. Translated in St. Petersburg Math. J. 5:4 (1994), 663-717.

[27] Pfannschmidt, C.: A generalization of the theorem of Hardy: a most general version of the uncertainty principle for Fourier integrals. Math. Nachr. 182 (1996), 317-327. 
[28] Wilcox C.H.: The synthesis problem for radar ambiguity functions. MRC Tech. Summary Report 157 (1960). Republished in Radar and Sonar part I (eds. R. Blahut, W. Miller and C. Wilcox). IMA Vol. Math. Appl. 32, 229260, Springer, New York, 1991.

[29] Wilczok, E.: New uncertainty principles for the continuous Gabor transform and the continuous wavelet transform. Doc. Math. 5 (2000), 201-226.

Recibido: 22 de marzo de 2001

Aline Bonami
Université d'Orléans
Faculté des Sciences
Département de Mathématiques, BP 6759
F 45067 Orléans Cedex 2, France
bonami@labomath. univ-orleans.fr
Bruno Demange
Université d'Orléans
Faculté des Sciences
Département de Mathématiques, BP 6759
F 45067 Orléans Cedex 2, France
bdemange@labomath.univ-orleans.fr
Philippe Jaming
Université d'Orléans
Faculté des Sciences
Département de Mathématiques, BP 6759
F 45067 Orléans Cedex 2, France
jaming@labomath. univ-orleans.fr

Research partially financed by : European Commission (TMR 1998-2001 Network Harmonic Analysis). 